\title{
AVALIAÇÃO DA FLORÍSTICA, DO PORTE E DA FITOSSANIDADE ATUAL DA ARBORIZAÇÃO DO PARQUE INTERNACIONAL EM SANT'ANA DO LIVRAMENTO/RIVERA, BRASIL/URUGUAI
}

\author{
Ana Claudia Bentancor Araujo ${ }^{\text {; }}$ Cibele Rosa Gracioli²; Edenir Luis Grimm³ ; Solon Jonas \\ Longhi $^{4}$
}

(recebido em 22.03.2011 e aceito para publicação em 15.03.2012)

\section{RESUMO}

O presente estudo teve como objetivo a avaliação quantitativa e qualitativa da composição vertical do Parque Internacional localizado no município de Sant'Ana do Livramento/BR e Rivera/UY, visando identificar, classificar e apresentar uma listagem das espécies arbóreas encontradas com suas respectivas famílias e número de ocorrência, além de avaliar o estado fitossanitário e a necessidade de manejo das árvores. Foram inventariados todos os indivíduos existentes com circunferência à altura do peito (CAP) maior ou igual a $15,7 \mathrm{~cm}$. Nessa etapa foram anotados e medidos dados referentes ao indivíduo como: CAP, altura total, origem, estado fitossanitário e necessidade de poda leve ou pesada. Foram inventariadas 300 árvores pertencentes a 24 famílias. Verificaram-se um baixo índice de espécies nativas existentes no Parque, 31\%, e um alto percentual de árvores exóticas, 69\%. Quanto ao número de indivíduos, destacam-se a família Cupressaceae, seguida pela Moraceae, Bignoniaceae, Oleaceae, Pinaceae e Fabaceae. Dentre os indivíduos encontrados as espécies Ligustrum japonicum seguida por Ficus microcarpa, Chamaecyparis lawsoniana e Handroanthus heptaphyllus foram as mais abundantes. Foram encontrados $18,27 \%$ indivíduos com o estado fitossanitário ruim e $81,73 \%$ indivíduos em bom estado. Dos indivíduos em mau estado fitossanitário destacam-se Magnolia grandiflora, Ligustrum japonicum e Handroanthus heptaphyllus que se encontram infestadas por ervade-passarinho.

Palavras-chave: silvicultura urbana, composição florística, paisagismo.

\footnotetext{
1 Engenheira Florestal, MSc, Professora do Instituto Federal Farroupilha. E-mail: bentancorana@gmail.com

2 Engenheira Florestal, Dra, Professora da Universidade do Pampa (Unipampa). E-mail: cibelegracioli@hotmail.com

${ }_{3}$ Engenheiro Agrônomo, Dr, Professor do Instituto Federal Farroupilha. E-mail: edenirgrimm@yahoo.com.br

${ }^{4}$ Engenheiro Florestal, Dr, Professor do Departamento de Engenharia Florestal da Universidade Federal de Santa Maria (UFSM). E-mail: longhi.solon@gmail.com
}

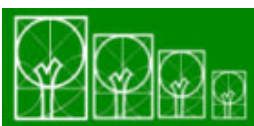

$\mathbf{S} \cdot \mathbf{B} \cdot \mathbf{A} \cdot \mathbf{U}$ Soc. Bras. de Arborização Urbana

REVSBAU, Piracicaba - SP, v.7, n.1, p. 112-125, 2012 


\section{FLORISTIC, SIZE, HEALTH AND ACTUAL PLANT PRESERVATION \\ EVALUATION OF TREES OF THE INTERNATIONAL PARK IN SANT'ANA DO \\ LIVRAMENTO/ RIVERA, BRAZIL/URUGUAY}

\section{ABSTRACT}

This study aimed to evaluate quantitative and qualitative vertical composition in International Park at Sant'ana do Livramento / BR and Rivera / UY, to identify, classify and present a species list with their families and occurrence number, and to evaluate the plant health and the management trees need. All existing individuals with circumference at breast height $(\mathrm{CBH})$ greater than or equal to $15.7 \mathrm{~cm}$ were inventoried. In this stage, measured data for the individuals were recorded, such as: $\mathrm{CBH}$, height, origin, plant health and the need for light or heavy pruning. In the Park 300 trees belonging to 24 families were surveyed. There has been a low rate of native species in the Park, $31 \%$ and a high percentage of exotic trees, $69 \%$. Regarding the number of individuals, Cupressaceae was the most important family, followed by Moraceae, Bignoniaceae, Oleaceae, Pinaceae and Fabaceae families. Among the individuals found the most frequent species were Ligustrum japonicum followed by Ficus microcarpa Chamaecyparis lawsoniana and Handroanthus heptaphyllus. A bad phytosanitary condition was observed in $18.27 \%$ of the individuals and $81.73 \%$ were in good condition. Regarding the individuals in worst phytosanitary condition Magnolia grandiflora, Ligustrum japonicum and Handroanthus heptaphyllus were the most infested by mistletoe.

Keywords: urban forestry, floristic composition, landscaping.

\section{INTRODUÇÃO}

No decorrer da história, são observadas, diferentes percepções sobre as árvores e suas funções no ambiente. Do extrativismo desordenado, nos últimos séculos, chegamos aos dias atuais numa situação de grande carência por áreas verdes, também no meio rural, mas principalmente no ambiente urbano (BRUN; BRUN, 2006). Conforme os mesmos autores, o desordenado crescimento das cidades causou problemas, como alta 
concentração de poluentes no ar, formação de ilhas de calor, poluição visual e sonora, devido à falta de planejamento neste crescimento.

Desta forma, a vegetação assume benefícios comprovados, como o bem estar propiciado ao homem; o melhor efeito estético; a sombra para os pedestres e veículos; barreira sonora, amenizando a poluição sonora; a redução do impacto da chuva e seu escorrimento superficial; e a preservação da fauna silvestre (PIVETTA; SILVA FILHO, 2002). Sendo assim, conforme Paiva e Gonçalves (2002), a vegetação urbana contribui para harmonia da paisagem quebrando a dureza e a rigidez do concreto, criando linhas mais suaves e naturais.

Locais com grande circulação de pessoas, quando bem arborizados, contam com a garantia de um ambiente onde há benefícios ecológicos, sociais e econômicos (MELO, 2005).

Para Demattê (1997) a arborização urbana resguarda sempre um pouco da flora original das cidades em suas praças, parques e locais de preservação. Praças são pontos de encontro cuja principal função é incentivar a vida comunitária; são áreas verdes com dimensões, em geral, entre $100 \mathrm{~m}^{2}$ e 10 ha. Porém, não se pode padronizar a praça, quanto ao tamanho, sem conhecer antes o seu entorno.

No entanto, uma arborização não planejada e realizada por pessoal inapto pode ter influência negativa direta em alguns elementos da organização urbana como redes de distribuição de energia elétrica e telefônica e sistemas de abastecimento de água e esgoto (MENESES et al., 2003). Portanto, para que as árvores introduzidas no espaço urbano não venham a causar problemas no futuro, deve haver um planejamento adequado considerando aspectos culturais e históricos da população local, com suas necessidades e aspirações, bem como o espaço físico disponível (PRASS, 2004).

Dantas et al. (2004) ressalta ainda que planejar a arborização é indispensável para o desenvolvimento urbano, para não acarretar prejuízos ao meio ambiente. Considerando que a arborização é um fator fundamental na salubridade ambiental, por ter influência direta sobre o bem-estar do homem em razão dos múltiplos benefícios que proporciona ao meio.

O Parque Internacional, pertence à cidade de Sant'Ana do Livramento/Brasil e à Rivera/Uruguai. Este foi criado em 26 de fevereiro de 1943 e constitui-se de uma área verde desfrutada em harmonia pela população das duas cidades vizinhas configurando-se como um caso único no mundo. O mesmo faz parte do conjunto arquitetônico-urbanístico e paisagístico dos dois municípios, tornando-se assim, de interesse ecológico, cientifico, turístico histórico, cultural e patrimonial.

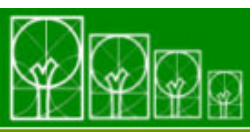

$\mathbf{S} \cdot \mathbf{B} \cdot \mathbf{A} \cdot \mathbf{U}$ Soc. Bras. de Arborização Urbana 
Neste contexto, estudos que visem avaliar a situação atual da flora do parque apresentam-se como subsídios para sua manutenção e enriquecimento.

Portanto, o presente estudo teve como objetivo a avaliação qualitativa e quantitativa da composição vertical do Parque Internacional, visando identificar, classificar e apresentar uma listagem das espécies arbóreas encontradas com suas respectivas famílias e número de ocorrência, além de avaliar o estado fitossanitário e a necessidade de manejo das árvores.

\section{MATERIAIS E MÉTODOS}

\section{Caracterização da área}

O trabalho foi realizado no Parque Internacional localizado entre os municípios de Sant'Ana do Livramento no Brasil (Latitude 30 48' 31" S e Longitude 5522' 33"O) e Rivera no Uruguai (Latitude 30 54' 20" S e Longitude 55 33'02”O).

O Parque possui aproximadamente 3 hectares. Os municípios encontram-se a uma altitude em torno de 208 metros acima do nível do mar sendo, o clima da região classificado como temperado úmido no verão, com temperatura média anual variando entre $12,1^{\circ} \mathrm{C} \mathrm{e}$ $18^{\circ} \mathrm{C}$ e a precipitação pluvial anual é em torno de $1.388 \mathrm{~mm}$ (MALUF, 2000). De acordo com Streck et al. (2008), nessa região ocorrem solos do tipo Argissolos Bruno-Acinzentados Alíticos Abruptos.

\section{Amostragem da Vegetação}

Foram inventariados todos os indivíduos existentes no Parque Internacional do município de Sant'Ana do Livramento/BR e Rivera/UY com circunferência à altura do peito (CAP) maior ou igual a $15,7 \mathrm{~cm}$. Nessa etapa foram anotados e medidos dados referentes ao indivíduo como: CAP, altura total mensurada com hipsômetro eletrônico-Vertex III, origem, estado fitossanitário e necessidade de poda leve ou pesada.

A origem foi classificada em nativa ou exótica. A altura foi classificada da seguinte maneira: pequenas $(H \leq 6 m)$, médias $(6,1 \mathrm{~m} \leq \mathrm{H} \leq 10 \mathrm{~m})$ e grandes $(\mathrm{H}>10 \mathrm{~m})$. Necessidade de manejo: foi avaliada para cada indivíduo, a necessidade ou não das seguintes práticas: poda leve (retirada de ramos que prejudicam a conformação natural das árvores), poda 
pesada (retirada de boa parte da copa, devido algum tipo de dano a outras espécies ou usuários do parque), controle fitossanitário (presença de pragas ou doenças visíveis por meio de danos nas diversas partes das árvores) e remoção do vegetal (indivíduos mortos ou com sintomas irrecuperáveis).

As espécies mais comuns e frequentes foram identificadas in loco, e as demais foram, posteriormente, identificadas no Herbário do Departamento de Ciências Florestais (HDCF) da Universidade Federal de Santa Maria/RS. A classificação das famílias botânicas segue as orientações do Angiosperm Phylogeny Group (APG II).

\section{RESULTADOS E DISCUSSÕES}

\section{Análise florística}

No Parque Internacional foram inventariadas 300 árvores, incluindo 1 morta e 1 não identificada, as espécies identificadas pertencem a 24 famílias destacando-se entre elas a Fabaceae, com 5 gêneros e 5 espécies e Pinaceae, com 5 gêneros e 5 espécies seguidas pelas famílias Arecaceae, com 4 gêneros e 5 espécies; Moraceae, com 2 gêneros e 4 espécies; Bignoniaceae, com 2 gêneros e 3 espécies e Cupressaceae, com 2 gêneros e 3 espécies (Tabela 1). Na composição florística do Parque, essas famílias são as mais importantes, estando representadas ao total por 20 gêneros e por 25 espécies.

Verificou-se um baixo índice de espécies nativas existentes no Parque, 25\%, e um alto percentual de árvores exóticas, 69\%.

O alto percentual de espécies exóticas encontrado no Parque Internacional não é o ideal, pois conforme os autores Sanchotene (1985), Blum et al. (2008) e Guia et al. (2008) deve-se priorizar o uso de espécies nativas. De acordo com estes pesquisadores o uso de nativas regionais, além de visar à preservação das espécies, ainda condiciona um equilíbrio fisiográfico maior, incorporando na paisagem urbana, elementos que propiciam sua integração com a paisagem da região.

O enriquecimento das áreas verdes urbanas com espécies nativas de caráter paisagístico representa um ganho em nível de valorização podendo contribuir, em parte, para a conservação das espécies, embora, possam sofrer diferenciações. Essas espécies devem apresentar resistência aos fatores de estresse do meio urbano, adaptabilidade 
climática, sobrevivência e desenvolvimento no local de plantio, além de resistência ao ataque de pragas e doenças (GUIA et al., 2008).

Dentre os indivíduos encontrados no Parque destacam-se a espécie Ligustrum japonicum, com 29 indivíduos, aparecendo em toda a extensão do Parque, seguida pela Ficus microcarpa, com 27 indivíduos, Chamaecyparis lawsoniana, com 23 indivíduos, Handroanthus heptaphyllus com 21 indivíduos. Destacou-se entre as exóticas em número de indivíduos a espécie Ligustrum japonicum, Ficus microcarpa e Chamaecyparis lawsoniana e entre as nativas a Handroanthus heptaphyllus, Caesalpinia peltophoroides e Vitex megapotanica (Tabela 1).

A área basal total encontrada no local foi de $32,7618 \mathrm{~m}^{2} /$ ha sendo a espécie Cunninghamia lanceolata a que apresentou o maior valor, seguida pela Chamaecyparis lawsoniana, Handroanthus heptaphyllus e Cupressus sempervirens (Tabela 1). Tais resultados são em decorrência dessas espécies serem representadas no Parque por indivíduos de grande porte.

De acordo com Santamour Júnior (2002), recomenda-se não exceder mais que $10 \%$ da mesma espécie, $20 \%$ de algum gênero e $30 \%$ de uma família botânica, pois, a maior diversidade de espécies de árvores na paisagem urbana se faz necessária para garantir o máximo de proteção contra pragas e doenças. Verificou-se que no Parque Internacional nenhuma espécie ultrapassa $10 \%$ em número de indivíduos (Tabela 1) assim como nenhuma família excede $30 \%$ do total de indivíduos da área (Figura 1). 
Tabela 1. Espécies de árvores encontradas no Parque Internacional em Sant'Ana do Livramento/BR e Rivera/UY, com sua família, nome científico, nome popular, número de indivíduos $(\mathrm{N})$, frequência, área basal e origem das espécies encontradas no Parque Internacional. Sant'Ana do Livramento/BR e Rivera/UY. 2011

Table 1. Tree species found in International Park in Sant'Ana do Livramento/BR and Rivera/UY with their families, scientific name, popular name, frequency, basal area and our origin. Sant'Ana do Livramento/BR and Rivera/UY. 2011

\begin{tabular}{|c|c|c|c|c|c|}
\hline Família/Nome científico & Nome popular & $\mathbf{N}$ & $\begin{array}{c}\text { Freq. } \\
(\%)\end{array}$ & $\begin{array}{c}\text { Área } \\
\text { basal } \\
\left(\mathrm{m}^{2} / \mathrm{ha}\right)\end{array}$ & Origem \\
\hline \multicolumn{6}{|l|}{ Anacardiaceae } \\
\hline Schinus molle & aroeira-salso & 5 & 1,7 & 0,363 & Nativa \\
\hline \multicolumn{6}{|l|}{ Araucariaceae } \\
\hline Araucaria heterophylla & araucária-heterophila & 1 & 0,3 & 0,137 & Exótica \\
\hline Araucaria angustifolia & pinheiro-brasileiro & 3 & 1 & 0,809 & Nativa \\
\hline \multicolumn{6}{|l|}{ Arecaceae } \\
\hline Butia capitata & butiá & 4 & 1,3 & 0,813 & Nativa \\
\hline Phoenix canariensis & palmeira-das-canarias & 4 & 1,3 & 0,921 & Exótica \\
\hline Siagrus romanzoffiana & jerivá & 1 & 0,3 & 0,204 & Nativa \\
\hline Washingtonia filifera & palmeira-de-saia & 3 & 1 & 0,953 & Exótica \\
\hline Washingtonia robusta & washingtônia & 1 & 0,3 & 0,081 & Exótica \\
\hline \multicolumn{6}{|l|}{ Apocynaceae } \\
\hline Nerium oleander & espirradeira & 3 & 1 & 0,048 & Exótica \\
\hline Thevetia peruviana & chapéu-de-napoleão & 3 & 1 & 0,202 & Exótica \\
\hline \multicolumn{6}{|l|}{ Bignoniaceae } \\
\hline Jacaranda mimosifolia & jacarandá-mimoso & 12 & 4 & 1,004 & Exótica \\
\hline Handroanthus heptaphyllus & ipê-roxo & 21 & 7 & 2,557 & Nativa \\
\hline Handroanthus chrysotrichus & ipê-amarelo & 4 & 1,3 & 0,052 & Nativa \\
\hline \multicolumn{6}{|l|}{ Cupressaceae } \\
\hline Cupressus macrocarpa & cipreste-de-monterei & 7 & 2,3 & 1,949 & Exótica \\
\hline Chamaecyparis lawsoniana & cipreste-escaravelho & 23 & 7,6 & 3,077 & Exótica \\
\hline Cupressus sempervirens & cipreste-mediterrâneo & 17 & 5,6 & 2,104 & Exótica \\
\hline
\end{tabular}




\section{Fabaceae}

Caesalpinia peltophoroides sibipiruna

Inga vera

inga-feijão

Robinia sp.

Schizolobium parahyba

guapuruvu

Tipuana tipu

tipuana

$\begin{array}{cccc}11 & 3,7 & 0,549 & \text { Nativa } \\ 8 & 2,7 & 0,570 & \text { Nativa } \\ 1 & 0,3 & 0,033 & \text { Exótica } \\ 2 & 0,7 & 0,233 & \text { Nativa } \\ 2 & 0,7 & 0,250 & \text { Exótica }\end{array}$

\section{Hamamelidaceae}

Liquidambar styracyflua

liquidambar

62

0,493 Exótica

\section{Lithraceae}

Lagerstroemia speciosa

resedá-gigante

$3 \quad 1 \quad 0,434 \quad$ Exótica

\section{Malvaceae}

Ceiba speciosa

paineira

$1 \quad 0,3$

$0,217 \quad$ Nativa

Magnoliaceae

Magnolia grandiflora

magnólia

62

0,309

Exótica

\section{Meliaceae}

Cedrela fissilis

cedro

$3 \quad 1$

0,040 Nativa

Myrtaceae

Psidium cattleianum

araça

$1 \quad 0,3$

0,014 Nativa

\section{Moraceae}

Ficus microcarpa

Morus nigra

Ficus elastica

Ficus luschnathiana

figueira-lacerdinha

$27 \quad 9$

0,703

Exótica

amora

93

0,428

Exótica

seringueira-de-jardim

0,578

Exótica

figueira

0,038

Exótica

Oleaceae

Fraxinus sp.

Ligustrum japonicum

ligustro

$\begin{array}{cccc}4 & 1,3 & 0,240 & \text { Exótica } \\ 29 & 9,6 & 0,871 & \text { Exótica }\end{array}$

Pinaceae

Cunninghamia lanceolata

pinheiro-chinês

Pinus taeda

pinus

11

3,7

4,365

Exótica

Cedrus sp.

3

1,336

Exótica

Thuja sp.

Juniperus chinensis

junípero-chinês

1

10

\section{Phytolacaceae}

Phytolacca dioica

umbu

31

0,147

Exótica

$10 \quad 3,3$

0,137

Exótica

$8 \quad 2,7$

0,299

Exótica

$1 \quad 0,3$

0,318 Nativa

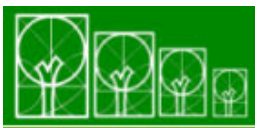




\section{Rhamnaceae}

Hovenia dulcis

uva-do-japão

$3 \quad 1$

0,193 Exótica

Rosaceae

Pirus sp.

Eriobotrya japonica

$\begin{array}{lllll} & 1 & 0,3 & 1,254 & \text { Exótica } \\ \text { ameixa } & 1 & 0,3 & 0,027 & \text { Exótica }\end{array}$

\section{Salicaceae}

Salix viminalis

vime

$1 \quad 0,3$

0,410

Exótica

Sterculiaceae

Brachychiton populneus

brachichita

$2 \quad 0,7$

0,446

Exótica

\section{Taxodiaceae}

\begin{tabular}{|c|c|c|c|c|c|}
\hline Cryptomeria japonica & pinheiro-japonês & 1 & 0,3 & 0,103 & Exótica \\
\hline \multicolumn{6}{|l|}{ Verbenaceae } \\
\hline Citharexylum montevidense & Tarumã-de-espinho & 3 & 1 & 0,034 & Nativa \\
\hline Vitex megapotanica & tarumã & 14 & 4,7 & 1,098 & Nativa \\
\hline \multicolumn{6}{|l|}{ Taxaceae } \\
\hline Taxus baccata L. & tejo & 8 & 2,7 & 0,434 & Exótica \\
\hline Morta & & 1 & 0,3 & 0,021 & \\
\hline Não identificada & & 1 & 0,3 & 0,866 & \\
\hline TOTAL & - & 300 & 100 & 32,7618 & - \\
\hline
\end{tabular}

Quanto ao número de indivíduos, destaca-se a família Cupressaceae, com 15,7\% de indivíduos, seguida pela Moraceae, com 13,7\% de indivíduos, Bignoniaceae, com 12,4\% de indivíduos, Oleaceae e Pinaceae, com $11 \%$ de indivíduos cada uma, Fabaceae, com $8 \%$ de indivíduos, Verbenaceae, com $5,7 \%$ de indivíduos, Arecaceae, com 4,7\% de indivíduos, que representam juntas $82,3 \%$ do total de indivíduos amostrados no local (Figura 1). 
Figura 1. Percentual de espécies, segundo a família, encontradas no Parque Internacional. Sant'Ana do Livramento/BR e Rivera/UY. 2011

Figure 1. Percentual of species in each family observed in International Park. Sant'Ana do Livramento/BR and Rivera/UY. 2011

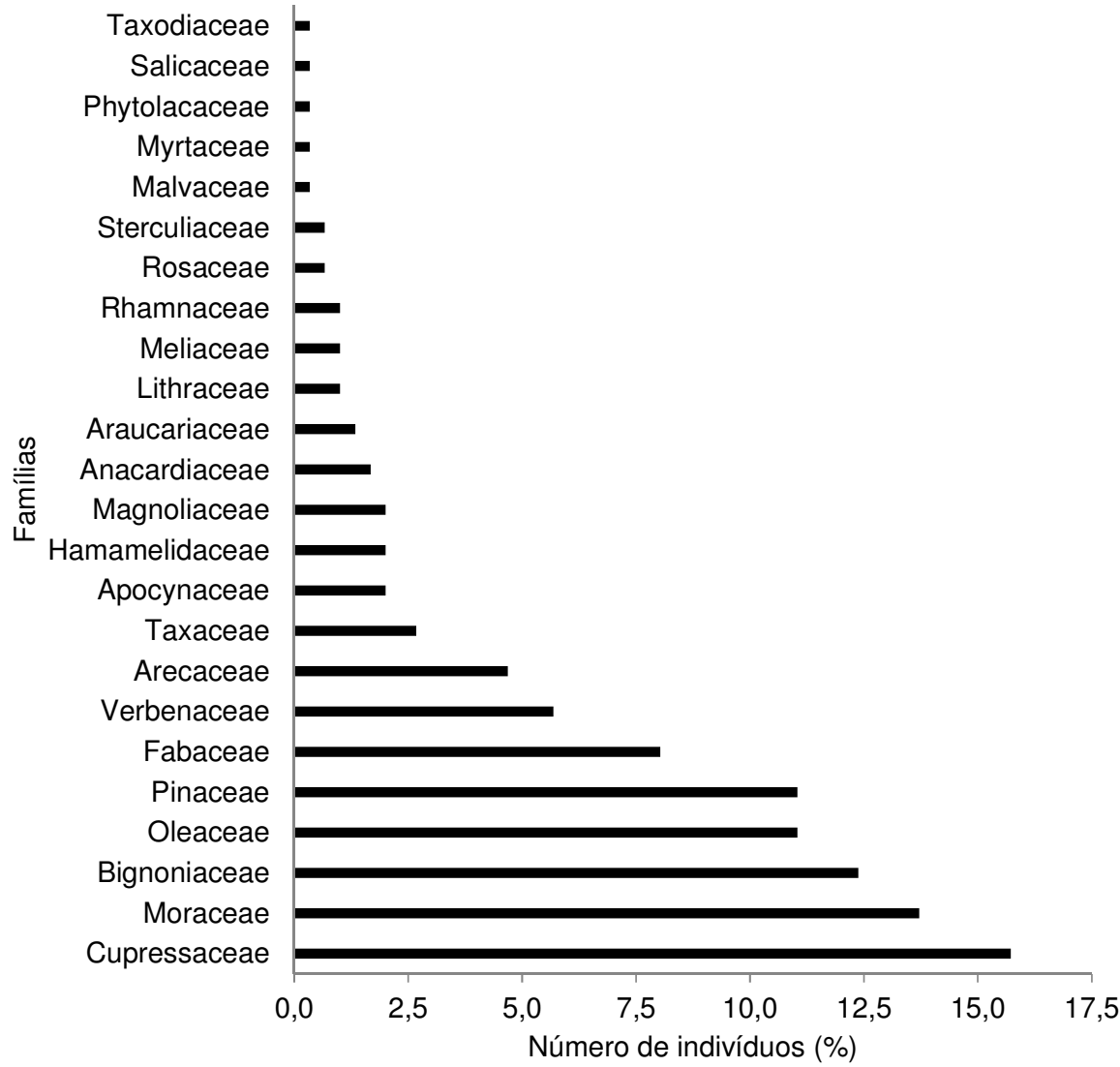

\section{Qualificação das árvores}

\section{Porte das árvores}

A avaliação do porte das árvores foi feita para possibilitar inferências sobre a sua composição etária, pois, não há informações confiáveis sobre a idade das árvores, ou época de plantio.

Após a avaliação total da área, observou-se que 32,9\% dos indivíduos foram considerados de grande porte (altura acima de 10 metros), 50,2\% possuem altura média (6,1 a 10 m) e 16,9\% das árvores são consideradas de baixa estatura. Entre as espécies de grande porte destacam-se: Cupressus macrocarpa, Araucaria angustifolia, Handroanthus heptaphyllus, Thevetia peruviana, Cunninghamia lanceolata, Pinus taeda, Brachychiton populneus, Chamaecyparis lawsoniana, Ficus microcarpa, Jacaranda mimosifolia, Liquidambar styracyflua, Cryptomeria japonica, Phoenix canariensis, Washingtonia robusta,

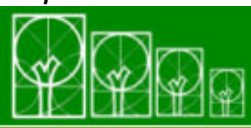


Chamaecyparis lawsoniana, Phoenix canariensis, Tipuana tipu, Schizolobium parahyba e Taxus baccata.

\section{Necessidade de manejo}

As árvores urbanas, de um modo geral, por estarem num habitat artificializado, estão mais sujeitas à ocorrência de pragas e doenças.

De acordo com o inventário qualitativo realizado na área foi possível constatar que $1,99 \%$ do total das árvores necessitam de poda pesada, sendo que esta deve ser executada na época de repouso vegetativo das árvores. De acordo com Guia et al. (2008) se os cortes não forem intensos, pode ser realizada em qualquer época do ano. As espécies de folhas perenes devem ser podadas no período que vai do término da frutificação à emissão de novos ramos. As espécies de folhas caducas devem ser podadas após perderem as folhas no fim da fase de repouso vegetativo, que pode ou não coincidir com o inverno.

No Parque foram encontrados $18,27 \%$ indivíduos com o estado fitossanitário ruim e 81,73\% indivíduos em bom estado. Dos indivíduos em mau estado fitossanitário destacamse Magnolia grandiflora, Ligustrum japonicum, Cupressus sempervirens, Liquidambar styracyflua, Ficus microcarpa, Handroanthus heptaphyllus, Schinus molle, Jacaranda mimosifolia, Hovenia dulcis, Tipuana tipu e Butia capitata (Figura 2) que se encontram infestadas por erva-de-passarinho (Sthruthanthus flexicaulis (Mart. ex Shult. F.).

Destacam-se as espécies $H$. heptaphyllus, que dos 21 indivíduos encontrados no Parque, 18 indivíduos estão infestados por erva-de-passarinho, L. japonicum, que dos 29 indivíduos, 12 também estão infectados com erva-de-passarinho. No entanto, o caso mais crítico foi verificado para as espécies $M$. grandiflora e Cedrus sp. em que todos os indivíduos catalogados estão infestados por hemiparasitas (erva-de-passarinho).

Guia et al. (2008) avaliando qualitativamente a composição vertical do Parque Antônio Pires de Campos, Cuiabá/MT, verificou que 34,82\% da composição arbórea do Parque estavam infestados por cupins, sendo a farinheira (Albizia hasslerii), pitombeira (Talisia esculenta) e jatobá (Hymenaea stigonocarpa) as espécies com maiores incidências. Notaram, também, que em $24,03 \%$ dos indivíduos, havia a presença de hemiparasitas (ervade-passarinho) em seus ramos. Conforme o mesmo autor alguns tipos de danos presentes nos vegetais iniciam-se com podas mal executadas, deixando exposta a área de corte, foco de entrada e desenvolvimento de pragas e doença. Com o tempo, essas doenças se alastram para outras partes da árvore, levando-as, às vezes, a morte prematura.

No Parque Internacional foi encontrado apenas um indivíduo morto ainda em pé em

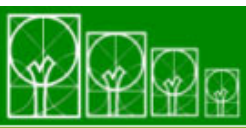

$\mathbf{S} \cdot \mathbf{B} \cdot \mathbf{A} \cdot \mathbf{U}$ Soc. Bras. de Arborização Urbana 
toda a extensão do mesmo, isso indica um índice de $0,33 \%$ do total de indivíduos catalogados.

Figura 2. Estado fitossanitário apresentado pelos indivíduos no Parque Internacional. Sant'Ana do Livramento, BR e Rivera/UY. 2011

Figure 2. Phytosanitary state presented by individuals at International Park. Sant'Ana do Livramento/BR e Rivera/UY. 2011
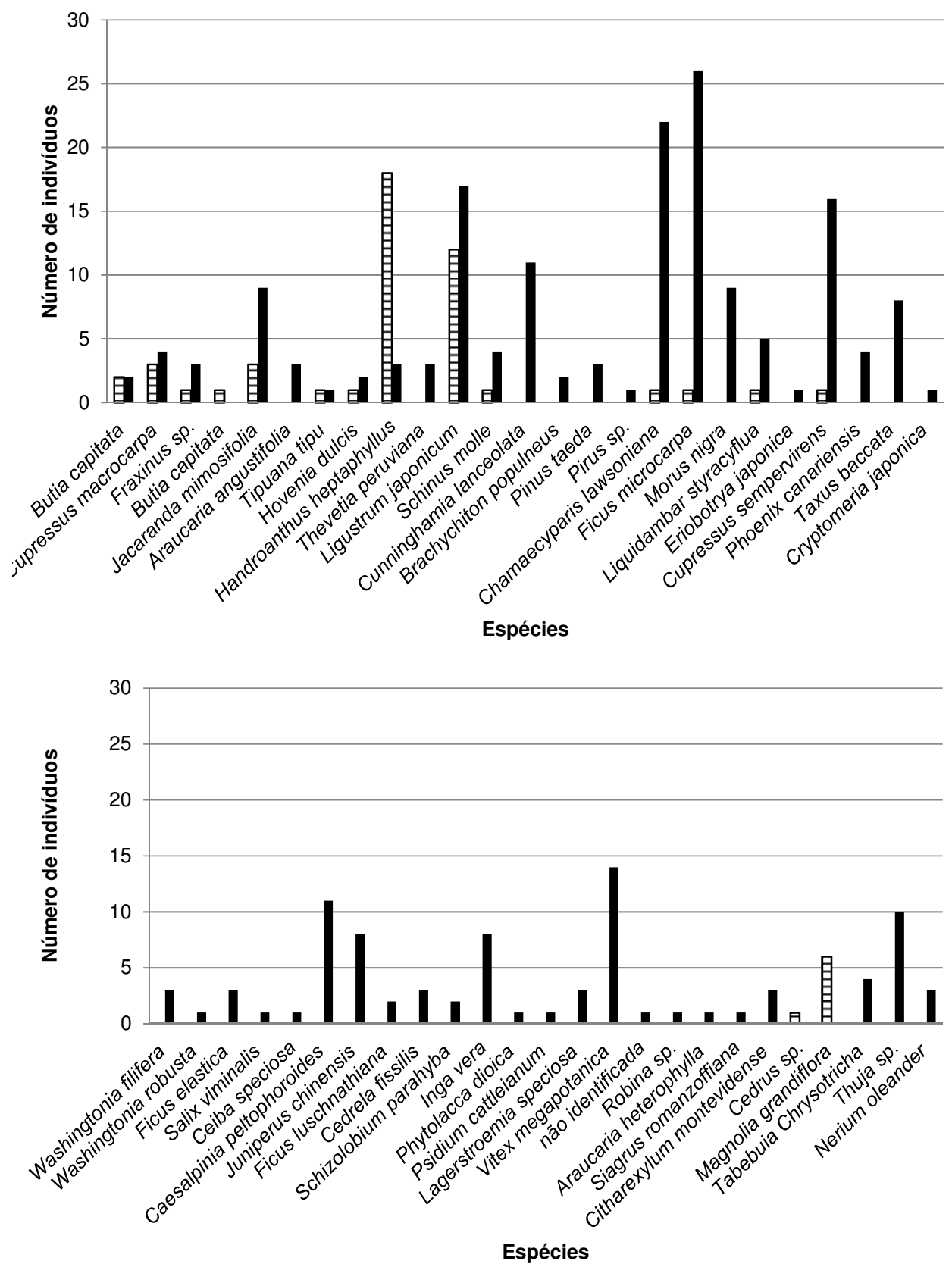

日Estado fitossanitário ruim घEstado fitossanitário bom 


\section{CONCLUSÕES}

A arborização do Parque Internacional possui uma satisfatória distribuição de gêneros e famílias, além de possuir uma adequada diversidade de espécies sendo que as mesmas apresentam, no geral, condições satisfatórias de sanidade. No entanto, merecem atenção especial quanto a sanidade as espécies Handroanthus heptaphyllus, Ligustrum japonicum, Magnolia grandiflora e Cedrus sp que estão infestadas de erva-de-passarinho o que pode levar as espécies a morte o que seria uma perda lastimável para a flora do Parque. Portanto, há a necessidade de manejo, como o corte dos galhos infestados na fase inicial, antes que tenha que se proceder uma poda mais drástica.

\section{REFERÊNCIAS}

BLUM, C. T.; BORGO, M.; SAMPAIO, A. C. F. Espécies exóticas invasoras na arborização de vias públicas de Maringá-PR. Revista da Sociedade Brasileira de Arborização Urbana, Piracicaba, v.3, n.2, p.78-97, jun. 2008.

BRUN, E. J. B.; BRUN, F. G. K. Arborização urbana \& qualidade de vida. Revista CREARS, Porto Alegre, n. 18, p. 27, fev. 2006.

DANTAS, C. I; DE SOUZA, C. M. C. Arborização urbana na cidade de Campina Grande- PB: Inventário e suas espécies. Revista de Biologia e Ciências da Terra, Campina Grande, v.4, n. 2, 2004.

DEMATTÊ, M.E.S.P. Princípios de paisagismo. Jaboticabal: Funep, 1997. 104p.

GUIA, G. H. da; ALBRETCH, J. M. F.; SOARES, T. S. TITON, M. Avaliação qualitativa das espécies arbóreas do Parque Antônio Pires de Campos em Cuiabá-MT. Revista da Sociedade Brasileira de Arborização Urbana, Piracicaba, v.3, n.3, p. 36-43, set. 2008.

MALUF, J. R. T. Nova classificação climática do Estado do Rio Grande do Sul. Revista Brasileira de Agrometeorologia, Santa Maria, v. 8, n. 1, p. 141-150. 2000.

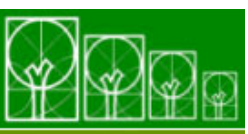


MENESES, C. H. S. G.; SOUSA, E. B. M.; MEDEIROS, F. P.; MENEZES, I. R.; ALBUQUERQUE, H. N.; SANTOS, L. Análise da arborização dos bairros do Mirante e Vila Cabral na cidade de Campina Grande - PB. Revista de Biologia e Ciências da Terra, Campina Grande, v.3, n.2. 2003.

MELO, E. F. R. Q.; Severo, B. M. A. Análise da Arborização do Campus da Universidade de Passo Fundo. In: IX Congresso Brasileiro de Arborização Urbana,1, Belo Horizonte, 2005. Anais. - . Belo Horizonte-MG.

PAIVA, H.N. de; GONÇALVES, W. Florestas urbanas: planejamento para melhoria da qualidade de vida. Viçosa: Aprenda Fácil, 2002. 177p. (Série Arborização Urbana, 2).

PIVETTA, K. F. L.; SILVA FILHO, D. F. Arborização Urbana - Boletim Acadêmico, Série Arborização Urbana. UNESP/FCAV/FUNEP: Jaboticabal-SP: 2002.

PRASS, C. H. Avaliação da arborização urbana na cidade de Quinze de Novembro - RS. Santa Maria, Universidade Federal de Santa Maria, Departamento de Ciências Florestais, p. 64, 2004. (Relatório de Estágio Curricular).

STRECK, E. V. et al. Solos do Rio Grande do Sul. 2. ed. Porto Alegre: EMATER/RS, 2008. $222 \mathrm{p}$.

SANTAMOUR JUNIOR, F. S. Trees for urban planting: diversity, uniformity, and common sense. In: METRIA CONFERENCE, 7., 1990, Lisle. Proceedings... Lisle: 1990. p. 57-66.

SANCHOTENE, M. M. C. Frutíferas nativas úteis à fauna na arborização urbana. Porto Alegre: Feplam, 1985. 311p. 